\title{
Thrombocytopenia in Confirmed HCV Cases
}

\author{
Warveen L Abdulkareem, ${ }^{1}$ Rozha A Abdullah, ${ }^{1}$ and Nawfal R Hussein ${ }^{1,}$ \\ ${ }^{1}$ Department of Internal Medicine, Azadi Teaching Hospital, College of Medicine, University of Duhok, Duhok, Kurdistan Region, Iraq \\ "Corresponding author: Nawfal R Hussein, Department of Internal Medicine, Azadi Teaching Hospital, College of Medicine, University of Duhok, Duhok, Kurdistan Region, \\ Iraq. E-mail: Nawfal.hussein@yahoo.com
}

Received 2016 September 05; Accepted 2016 September 06.

Keywords: HCV, Thrombocytopenia, Infection

\section{Dear Editor,}

We have read, with great interest, the study conducted by Bano et al. (1) in your journal. The aim of their study was to investigate the prevalence of thrombocytopenia in HCV patients without cirrhosis and splenomegaly. They recruited 30 patients for the study, and found the prevalence of thrombocytopenia to be $43.3 \%$. Moreover, they concluded that thrombocytopenia was of moderate frequency in $\mathrm{HCV}$ participants. Undoubtedly, $\mathrm{HCV}$ is a public health problem worldwide, especially in developing countries $(2,3)$. The classical treatment of HCV is challenging and fraught with poor tolerability and side effects. A major breakthrough occurred in managing HCV with new direct acting antiviral regimens with less side effects and more tolerability. However, the use of such new regimens is restricted due to their high price; therefore, classification of the patients is necessary (4). Here comes the importance of thrombocytopenia, as it can be considered a contraindication for the classical regimen $(5,6)$. Any patient with thrombocytopenia might be eligible for the new expensive medications. Hence, it might be important for the public health planners to estimate the prevalence of thrombocytopenia in HCV. Furthermore, thrombocytopenia in chronic HCV patients is associated with bleeding tendency, and it is a poor prognostic factor. However, it is important to clarify that HCV-antibody positivity does not indi- cate current infection, but shows an exposure to the virus. To a large extent, the diagnosis of HCV depends on the PCR positivity. Thus, any study targeting HCV patients should include patients with confirmed PCR results. Moreover, a larger sample size is needed for prevalence study. Therefore, the recruitment of a larger sample with a confirmed current HCV infection is highly recommended.

\section{References}

1. Bano S, Qureshi J, Raza A, Zafar Hashmi K. Thrombocytopenia as a Clinical Manifestation of Hepatitis C Among Patients With a Positive AntiHCV Test. Int J Infect. 2016;3(3):35094. doi: 10.17795/iji-35094.

2. Papatheodoridis G, Hatzakis A. Public health issues of hepatitis $C$ virus infection. Best Pract Res Clin Gastroenterol. 2012;26(4):371-80. doi: 10.1016/j.bpg.2012.09.012. [PubMed: 23199497].

3. Hussein NR, Haj SM, Almizori LA, Taha AA. The Prevalence of Hepatitis $B$ and C Viruses Among Blood Donors Attending Blood Bank in Duhok, Kurdistan Region, Iraq. Int J Infec. 2016;In press(In press).

4. Shafran SD. The hepatitis $C$ genotype 1 paradox: cost per treatment is increasing, but cost per cure is decreasing. Can J Gastroenterol Hepatol. 2015;29(1):46-8. doi: 10.1155/2015/216395. [PubMed: 25706574].

5. Hussein NR. Sofosbuvir and Ribavirin to Treat Hepatitis C Virus Genotype 4 Infection in a Patient With Diabetes and Low Platelet: A Case Report. Int J Infec. 2016 doi: 10.17795/iji-41216.

6. Danish FA, Koul SS, Subhani FR, Rabbani AE, Yasmin S. Considerations in the management of hepatitis C virus-related thrombocytopenia with eltrombopag. Saudi J Gastroenterol. 2010;16(1):51-6. doi: 10.4103/1319-3767.58772. [PubMed: 20065578]. 